\title{
Laser capture microscopy
}

\author{
S Curran, J A McKay, H L McLeod, G I Murray
}

\begin{abstract}
Human tissues are composed of complex admixtures of different cell types and their biologically meaningful analysis necessitates the procurement of pure samples of the cells of interest. Many approaches have been used in attempts to overcome this difficulty, including a variety of microdissection methods. This review concerns a recent advance in microdissection techniques, namely laser capture microdissection (LCM). The principle underlying this technique is outlined, and practical issues pertaining to LCM are considered. In addition, the literature relating to LCM is reviewed, with examples of research applications of this technique being outlined.

(f Clin Pathol: Mol Pathol 2000;53:64-68)
\end{abstract}

Keywords: tissue microdissection; laser microscopy

The pace of development of molecular analytical techniques has been rapid in recent years. High throughput methods such as DNA microarrays ("DNA chip technology") and proteomics have enormous potential for revolutionising the study of molecular events associated with physiological and pathological processes. ${ }^{12}$ Although the technology has become increasingly sophisticated, the accuracy of the results obtained is still largely influenced by the selection of appropriate study material in the first instance. For many types of assays, the use of homogenised tissue samples can result in an "averaging out" of the heterogeneous elements of the sample. Human tissues are composed of admixtures of many different cell types, from which it is necessary to obtain a pure sample of the cells of interest to acquire meaningful data. The concept of tumour heterogeneity is increasingly being recognised, and it will be important to define and analyse separately subpopulations of cells within a neoplasm to identify properties that might be unique to them. Such properties, which might otherwise go undetected, could provide crucial insights into cancer cell biology.

Many techniques have been used in attempts to isolate pure cell populations, including cell culture studies, xenografts, flow cytometry, and various microdissection methods. Cell culture studies have been used extensively in the study of cell biology. However, cultured cells might not accurately reflect the biological characteristics of the cells from which they are derived because they are subject to different environmental factors. Surprisingly few studies have investigated this issue. Zhang et al reported differences in gene expression between primary colon cancer cells and cell lines. ${ }^{3}$ Xenografts have the advantage of being self replicating, but are expensive, time consuming, and require a high degree of expertise. Flow cytometry plays an important role in the study of many disease processes and has been used to separate cell populations from heterogeneous tissues. This technique requires the formation of a suspension of cells, which limits its usefulness for the study of solid tissues and tumours. In addition, a specific marker must be available for selection of the cell population under investigation.

A variety of microdissection techniques have been used in attempts to overcome the heterogeneity of tissues. Initially, these involved the use of a needle to scrape off the tissue of interest from a histological section. ${ }^{4}$ These methods tended to be slow, tedious, and highly operator dependent. Modifications included negative ablation, in which the cells of interest were coated with a pigment, the section then being exposed to ultraviolet light, destroying DNA in the uncoated cells. ${ }^{5}$

The aim of this review is to outline the principles underlying laser capture microdissection (LCM), give an overview of some research applications to date, and suggest possible future uses of this technique. We will focus on practical aspects of LCM, including tissue preparation, the technique of microdissection itself, and the types of analyses that can be performed on microdissected tissue, including high throughput methodologies.

\section{The principle of LCM}

LCM is a recently developed technique that permits the rapid and reliable procurement of pure populations of cells from tissue sections, in one step, under direct microscopic visualisation. This method was developed at the National Cancer Institute (NCI) specifically for the analysis of populations of cells from tumours and a commercial instrument, developed jointly by NCI and Arcturus Engineering Incorporated (Mountain View, California, USA), became available within 12 months of the first publication describing its use. ${ }^{6}$

The principle underlying LCM is simple (fig 1). A histological section is placed on the stage of the specially designed microscope and the pathologist selects the area of interest. A transparent ethylene vinyl acetate layer is apposed to the section and a low power infrared laser beam is directed at the cells of interest. When the operator activates the laser beam, the film directly above the targeted area transiently melts and the long chain polymers in the film surround and tightly hold the cells, which remain embedded in the film after its removal from the section. In the current commercially available version of the LCM, the size of the laser beam spot can be selected at $30 \mu \mathrm{m}$,
Accepted for publication 20 January 2000 

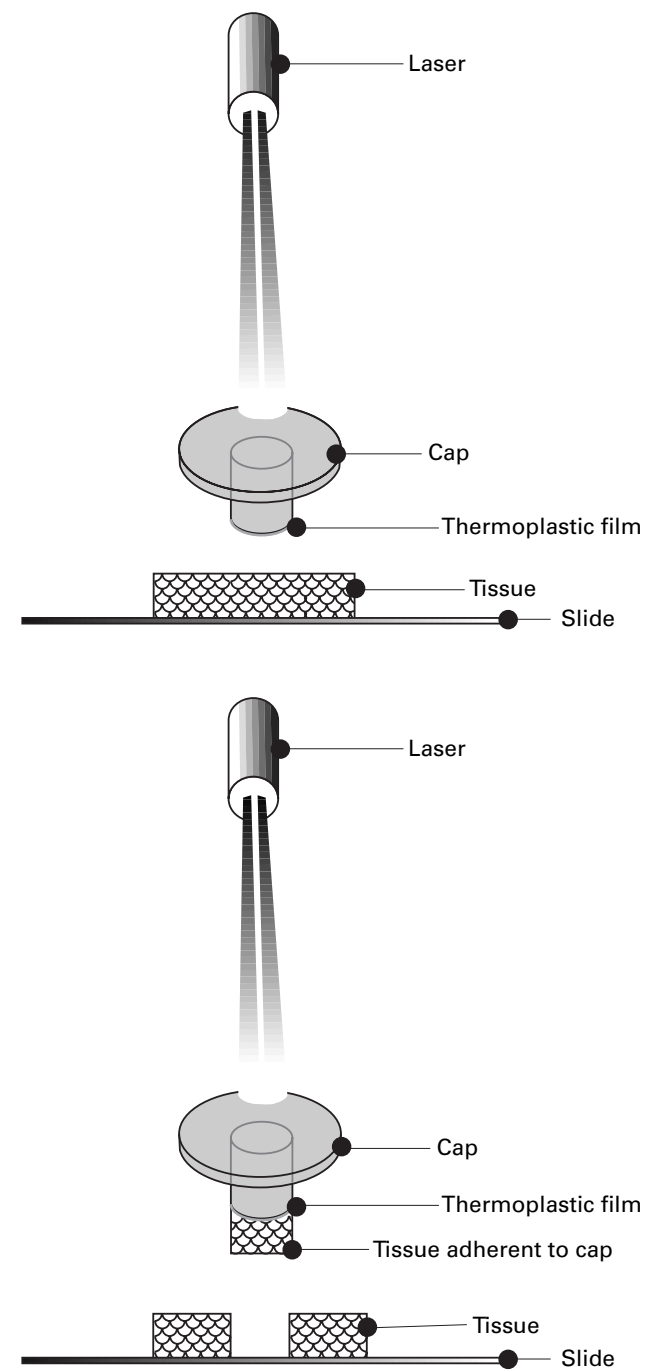

Figure 1 The principle of laser capture microdissection. The laser beam transiently melts the thermoplastic film coating the cap, causing the film to adhere to the chosen cells. On removal of the cap from the slide, the chosen tissue remains adherent to the cap.

$15 \mu \mathrm{m}$, or $7.5 \mu \mathrm{m}$, facilitating the dissection of groups of cells or even single cells. The technique can be used on formalin fixed, paraffin wax embedded sections, allowing the use of archival pathological material. In addition, frozen sections of tissue and cytological speci- mens are also suitable for laser microdissection. The technique causes no apparent alteration to the morphology of the chosen cells, which can be directly visualised after microdissection (fig 2). The cells of interest can be chosen on the basis of their morphology, immunohistochemical phenotype, or even, theoretically, on their genotype, using in situ hybridisation methods.

\section{Technical considerations}

LCM is a powerful tool that can be used to obtain exceptionally pure samples for use in many molecular analytical techniques. However, the mode of preparation of the tissue sections has to be taken into account with regard to which molecular analyses are feasible and will provide reliable and meaningful results. The effects of crosslinking fixatives (aldehydes) and precipitative fixatives (ethanol and acetone) on RNA extraction and amplification from microdissected tissue have been compared. Unsurprisingly, precipitative fixatives consistently produced more polymerase chain reaction (PCR) amplification product than crosslinking fixatives. The quantity of amplification product obtained was greater from frozen tissue than from paraffin wax embedded tissue. $^{7}$

Recognising that formalin fixation has a predictable adverse effect on nucleic acids, Maitra and co-workers devised a method of enrichment of epithelial cells for use in conjunction with LCM, which they termed EASI (epithelial aggregate separation and isolation). In their illustrative paper, fresh samples of carcinomas (breast, lung, colon, and prostate) were used. LCM was performed in the usual way and molecular analyses were then carried out, using PCR based assays. In the case of a colon cancer, amplification products were compared between formalin fixed tissue, methanol fixed "EASI-prep" tissue, frozen tissue, and a cancer cell line. DNA from the formalin fixed tissue failed to amplify products beyond $268 \mathrm{bp}$, whereas DNA from the EASI-prep sample amplified products up to $989 \mathrm{bp}$. The DNA obtained was used for loss of heterozygosity studies, single strand conformational polymorphism assays, and sequencing studies. With regard to RNA preservation, RNA suitable for
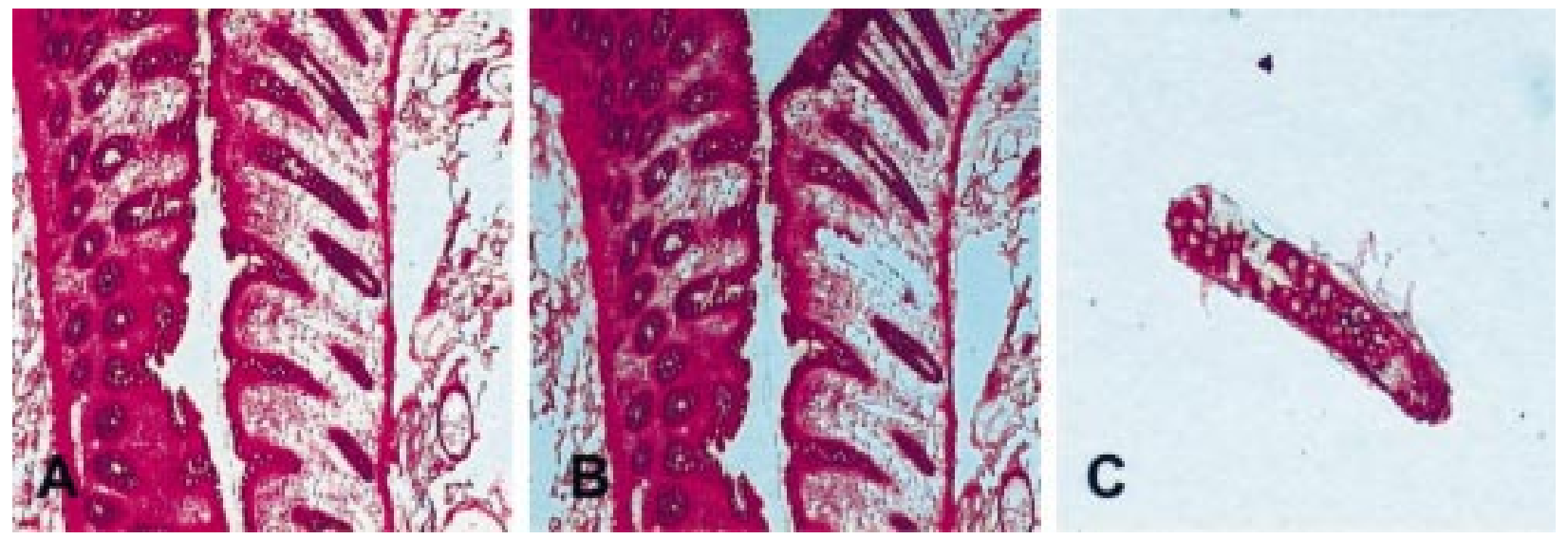

Figure 2 Frozen section of normal colon, stained with haematoxylin and eosin. (A) Before microdissection and (B) after microdissection; (C) captured tissue adherent to the cap. 
the reverse transcription polymerase chain reaction (RT-PCR) was obtained consistently from EASI-prep slides that had been stored at room temperature for up to seven days. Consistent and reproducible cDNA amplification (product sizes, 340-515 bp) was observed. ${ }^{8}$

We routinely use $7 \mu \mathrm{m}$ thick sections of frozen tissue for performing LCM, but sections of between 5 and $20 \mu \mathrm{m}$ in thickness can be used. In general terms, the thicker the tissue section, the greater the laser "power" required to dissect cells.

The tissue sections are mounted on plain, untreated glass slides. The use of adhesives or charged slides increases the strength of adhesion of the section to the slide, which interferes with tissue capture. In both our experience and the findings of others, LCM works best with stained and dehydrated sections. Any moisture, even a trace amount, appears to inhibit transfer of cells to the transfer film.

\section{Disadvantages of LCM}

LCM is a major advance in microdissection techniques, but a number of potential disadvantages exist.

The microscope and accompanying computer hardware and software require an initial capital outlay of approximately £70-90 000, and the cost of specially designed caps (approximately $£ 3$ for each cap) for capturing the tissue must also be considered.

Microscopic visualisation is less than perfect because of the absence of mounting medium and a coverslip. The Pixcell II model is equipped with a diffusion filter that diffracts light passing through the cap, facilitating clearer visualisation of the tissue. When the identification of cell types for microdissection is performed by an experienced histopathologist, the less than optimum visualisation is not a problem. The issue of impaired microscopic visualisation is common to most, if not all, microdissection methods.

Although LCM is considerably faster than other microdissection methods, the amount of researcher time required to carry out microdissection can be considerable, particularly where a large number of tissue samples is involved. However, this has to be balanced against the purity of the tissue sample obtainable using LCM, which is not feasible with previous methods of microdissection.

\section{Applications of LCM}

GENETIC ANALYSES

In the first paper describing LCM, EmmertBuck and co-workers demonstrated the validity of using the technique in conjunction with a variety of genetic analyses. Among these were loss of heterozygosity studies in several different tumour types, including breast cancer, (the BRCA1 gene), prostate cancer (chromosome $8 \mathrm{p}$ ), oesophageal cancer (p16 gene), and a multiple endocrine neoplasia associated gastrinoma (the putative MEN-1 gene). ${ }^{6}$ In addition, this group of investigators used LCM to dissect samples of premalignant lesions and normal epithelium from breast and prostate tissue sec- tions. Thus, this technique can facilitate comparative studies between normal, premalignant, and malignant cells from the same individual. This type of approach should prove valuable in the elucidation of the genetic steps along the pathway from normal to malignant cells.

There is a steadily growing number of reports of laser capture microscopy being used in conjunction with various genetic analyses. Although the technique has been used extensively in cancer research, it also lends itself to the study of any complex tissue. For example, in an interesting experiment on the effect of the adipostatic hormone leptin on adrenal function, laser capture microdissection was used to separate adrenocortical from adrenomedullary cells and these cells were analysed separately. Using RT-PCR, mRNA for the leptin receptor was identified in both types of cells. Immunohistochemistry revealed strong positivity for receptor protein in the cortical cells, with weaker staining of the adrenomedullary cells. These investigators demonstrated leptin induced inhibition of the production of aldosterone, cortisol, and dehydroepiandrosterone by cortical cells. In contrast, leptin did not have a significant effect on catecholamine production by medullary chromaffin cells. ${ }^{9}$ Similarly, laser microscopy has facilitated the study of the expression of prolactin receptors in adrenocortical and adrenomedullary cells isolated by LCM. In the study of Glasow et al, it was shown that cortical but not adrenomedullary cells expressed mRNA for the prolactin receptor. ${ }^{10}$

LCM can be combined with immunohistochemistry in the study of subpopulations of cells of a specific immunophenotype. This was illustrated by Fend et al, who used a rapid method of immunohistochemistry to stain distinct populations of either epithelial or lymphoid cells from lymph node, breast, and prostate tissue. A rapid method (total processing time of 12-25 minutes) was used to minimise degradation of nucleic acid during the immunohistochemical procedure. Laser capture was then used to microdissect immunophenotypically distinct populations of cells and RT-PCR for $\beta$ actin, CD4, or CD19 mRNA was performed on the microdissected cells. Amplification of $\beta$ actin was successful from less than 200 cells and amplification of CD4 or CD19 was achieved using less than 1000 cells. ${ }^{11}$

A recent paper used LCM in conjunction with immunohistochemistry to define anterior pituitary cells with a distinct immunohistochemical profile. RT-PCR was performed on single cells and groups of cells, and the authors reported accurate analysis of mRNA, even using a single cell. ${ }^{12}$ Cells that stained positively for prolactin or adrenocorticotrophic hormone (ACTH) were identified by immunohistochemistry, and were selectively microdissected. Subsequent RNA analysis (using RT-PCR) revealed that prolactin cDNA was only obtained from prolactin positive cells and not from those cells staining positively with antiACTH antibody. Accordingly, cDNA for 
pro-opiomelanocortin (POMC, a precursor of ACTH) was only detected from ACTH positive cells and not prolactin positive cells. Normal rat hepatocytes did not contain detectable amounts of either prolactin or POMC mRNA. A housekeeping gene (hypoxanthine phosphoribosyl transferase), used as a positive control, showed amplification of mRNA in all samples. ${ }^{12}$

Laser capture microscopy has been applied to the study of composite B cell non-Hodgkin's lymphomas, which are rare neoplasms containing admixtures of morphologically distinct cell populations possessing differing immunophenotypes. Fend et al used immunohistochemistry for CD5, CD10, CD43, and cyclin D1 to classify three examples of this rare lesion (case 1, mantle cell lymphoma and follicular lymphoma; case 2, follicular lymphoma and small lymphocytic lymphoma; and case 3, mantle cell lymphoma and chronic lymphocytic leukaemia). PCR analyses of DNA isolated from whole tissue sections failed to reveal evidence of polyclonality, but molecular analysis of microdissected cells selected on the basis of their immunophenotypes revealed that these lesions are frequently biclonal. The authors performed PCR amplification and sequenced clonal immunoglobulin heavy chain $(\operatorname{IgH})$ gene rearrangements and oncogene rearrangements. For each case, sequencing uncovered unrelated clonal rearrangements in the component tumour parts, suggesting that these lesions are truly biclonal. In addition, a bcl-2 rearrangement was identified exclusively in the follicular lymphoma area of one of the tumours. ${ }^{13}$

A recent report from the NCI poses an interesting hypothesis regarding the pathogenesis of Hodgkin's disease in immunosuppressed patients. Kumar and co-workers used CD30 immunohistochemistry to identify Reed-Sternberg cells in two cases of primary gastrointestinal Hodgkin's disease in patients with a history of chronic inflammatory bowel disease, treated with immunosuppressive agents. Reed-Sternberg cells were dissected using LCM and assessed for clonality using a PCR based assay for IgH gene rearrangement. Although one of the tumours appeared to be monoclonal, the other showed evidence of polyclonality. The authors suggest that the polyclonal case might be an example of a lymphoproliferative process, exhibiting the morphological and immunohistochemical appearances of Hodgkin's disease. An analogy is drawn with plasmacytic and polymorphic B cell hyperplasia arising in a post-transplant setting. It is possible that clonality in Hodgkin's disease will prove to be of prognostic importance. ${ }^{14}$

LCM has been used to extract individual tumour cells from a basal cell carcinoma of the skin with subsequent analysis of p53 mutations by sequencing of PCR products. Identical mutations were identified in cells from different parts of the tumour. In addition, when p53 immunoreactivity was correlated with mutational analysis, cells of differing immuno- phenotype were found to possess identical mutations. ${ }^{15}$

LCM is also being used in the cancer genome anatomy project, where two types of cDNA libraries are being generated: microdissected libraries and bulk tissue libraries. The aim is to catalogue the genetic profile of pure populations of tumour cells at different stages of tumour development. ${ }^{16}$

MICROARRAYS

The possibility of exploiting new, high throughput techniques in combination with LCM has been explored. In a paper using laser microscopy together with DNA microarray technology to compare gene expression between large and small neurones in the dorsal root ganglia, good reproducibility of RNA amplification was found between individual captures. In this study, 1000 neurones were used for each capture, but the authors found similar results using 500 cells, and suggest that far fewer cells might be required. Five mRNAs were preferentially expressed in small neurones - a fatty acid binding protein, a sodium voltage gated channel, calcitonin gene related peptide, phospholipase $\mathrm{C} \delta 4$, and annexin V. In large neurones, neurofilaments NF-L and NF-H, as well as the $\beta 1$ subunit of voltage gated sodium channels were preferentially expressed. These investigators used in situ hybridisation to confirm their findings. ${ }^{17}$

There has been a recent report of the use of LCM in conjunction with high throughput cDNA microarrays in the study of normal, malignant, and metastatic breast cells from the same individual. ${ }^{18}$ This type of approach might enable us to trace genetic alterations occurring within an individual during the development of neoplasia.

\section{PROTEIN ANALYSES}

The original paper describing LCM illustrated the use of the technique in acquiring dissected prostate cancer tissue for use in gel zymography experiments to detect the activity of matrix metalloproteinase $2 .^{6}$

More recently, a preliminary study by Banks et al assessed the feasibility of using LCM to obtain samples for analysis of the protein complement of the genome (proteomic analysis). The authors dissected renal tissue samples and observed identical mass spectrometric sequencing profiles for a number of selected proteins. This group found little change in proteins after the application of LCM to the tissue. $^{19}$

Using LCM, we have microdissected pure samples of normal colonic epithelium and colon cancer cells and performed two dimensional gel electrophoresis, followed by mass spectrometry. Our preliminary results show selective loss of non-epithelial proteins in microdissected samples as compared with whole tissue samples.

The proteomic approach promises to allow the identification of qualitative or quantitative differences in proteins between normal and diseased cells. As well as providing insights into 
pathogenesis, such information might yield new screening or therapeutic targets.

\section{Other laser based microdissection systems}

In addition to LCM, alternative laser based microdissection systems have been developed recently. Schütze and Lahr describe a microdissection system that does not require any contact to obtain the cells. The cells of interest were isolated using a low power laser to create a gap between these cells and surrounding cells. The power of the laser was then increased and used to "catapult" the microdissected cells into the cap of a microfuge tube for subsequent analysis. Using RT-PCR, they looked for point mutations in codons 12 and 13 of the Ki-ras oncogene in colorectal cancer cells. Mutations within codon 12 , but not 13 , were found in all the tumour samples analysed. These investigators showed that a single cell, derived from archival material, is sufficient for gene analysis. $^{20}$

In the system described by Fink et al, the cells that are not of interest to the investigator are photo ablated by an ultraviolet light laser operated under microscope control, and the remaining cells of interest acquired using a micromanipulator directed sterile needle. This method is potentially more time consuming than LCM in acquiring the cells of interest, but has the advantage that cells do not have to be retrieved from a polymer film. Alveolar macrophages were microdissected from intact animal lung tissue and the expression of tumour necrosis factor $\alpha(\mathrm{TNF}-\alpha)$ in the microdissected cells was analysed using real time quantitative RT-PCR. Analysis of TNF- $\alpha$ expression was successful using as few as 20 cells. $^{21}$

Using the same ultraviolet microbeam system as Fink et al, together with a refinement of whole genome amplification, Dietmaier et al sequenced exons 7 and 8 of the p53 gene in a series of breast carcinomas. They documented reliable procurement of material suitable for studies of microsatellite instability, loss of heterozygosity, and mutational analysis, even at the single cell level. ${ }^{22}$

Another group of workers has reported the use of the ultraviolet microbeam microdissection method in combination with laser pressure catapulting to isolate single chromosomes for the development of chromosome specific paint probes. $^{23}$

\section{Future developments}

Laser capture microdissection is still undergoing refinements, with the development of a prototype capable of precisely targeting single cells using short pulses of a laser beam of $6 \mu \mathrm{m}$ diameter. ${ }^{22}{ }^{23}$ The LCM system would lend itself to automation - for example, by adding a motorised $\mathrm{x}$, $\mathrm{y}$ stage to the microscope, the operator could define the area(s) to be dissected, with the actual tissue dissection being performed automatically.
In conclusion, laser capture microscopy is a practical method for the rapid and efficient isolation of specific populations of cells, free of contaminants and amenable to a variety of molecular analyses. This technology has now opened the door to enhancing our understanding of the molecular mechanisms regulating cellular functioning in normal and disease states.

The laser capture microscope was purchased by an equipment grant from the Faculty of Medicine and Medical Sciences, University of Aberdeen. This research has been supported by the Aberdeen colorectal cancer initiative (steering committee: J Aberdeen colorectal cancer initiative (steering committee: J
Cassidy, HL McLeod, GI Murray, N Haites, J Little, and WT Cassidy, HL McLeod, GI Murray, N Haites, J Little, and WT
Melvin), through a University of Aberdeen Development Trust Melvin), through a University of Aberdeen Development
grant. Mr G Stables provided medical illustrative support.

1 Shibata D. Pattern recognition and arrays. Am f Pathol 1999;154:979-80.

2 Blackstock WP, Weir MP. Proteomics: quantitative and physical mapping of cellular proteins. Trends Biotechnol
phestive 1999;17:121-7.

3 Zhang L, Zhou W, Velculescu VE, et al. Gene expression profiles in normal and cancer cells. Science 1997;276:1268 72

4 Going JJ, Lamb RF. Practical histological microdissection for PCR analysis. $\mathcal{F}$ Pathol 1996;179:121-4.

5 Shibata D, Hawes D, Li ZH, et al. Specific genetic analysis of microscopic tissue after selective ultraviolet radiation fractionation and the polymerase chain reaction. Am $\mathcal{F}$ Pathol 1992;141:539-43.

6 Emmert-Buck MR, Bonner RF, Smith PD, et al. Laser capture microdissection. Science 1996;274:998-1001.

7 Goldsworthy SM, Stockton PS, Trempus CS, et al. Effects of fixation on RNA extraction and amplification from laser capture microdissected tissue. Mol Carcinog 1999;25:8691.

8 Maitra A, Wistuba II, Virmani AK, et al. Enrichment of epithelial cells for molecular studies. Nat Med 1999;5:459-63.

Glasow A, Haidan A, Hilbers U, et al. Expression of Ob Glasow A, Haidan A, Hilbers U, et al. Expression of Ob
receptor in normal human adrenals: differential regulation of adrenocortical and adrenomedullary function by leptin. f Clin Endocrinol Metab 1998;83:4459-66.

10 Glasow A, Haidan A, Gillespie J, et al. Differential expression of prolactin receptor (PRLR) in normal and tumorous adrenal tissues: separation of cellular endocrine compartments by laser capture microdissection. Endocr Res 1998;24:857-62.

11 Fend F, Emmert-Buck MR, Chaqui R, et al. ImmunoLCM: laser capture microdissection of immunostained frozen sections for mRNA analysis. Am f Pathol 1999;154:61-

12 Jin L, Thompson CA, Qian X, et al. Analysis of anterior pituitary hormone mRNA expression in immunophenotypically characterized single cells after laser capture microdissection. Lab Invest 1999;79:511-12.

13 Fend F, Quintanilla-Martinez L, Kumar S, et al. Composite low-grade B-cell lymphomas with two immunophenotypically distinct cell populations are true biclonal lymphomas: a molecular analysis using laser capture microdissection. Am f Pathol 1999;154:1857-66.

14 Kumar S, Fend F, Quintanilla-Martinez L, et al. EpsteinBarr virus-positive primary gastrointestinal Hodgkin's disease. Am F Surg Pathol 2000;24:66-73.

15 Ponten F, Williams C, Ling G, et al. Genomic analysis of single cells from human basal cell cancer using laserassisted capture microscopy. Mutat Res 1997;16:45-55.

16 Cancer Genome Anatomy Project. http:// www.ncbi.nlm.nih.gov/CGAP.

17 Luo L, Salunga RC, Guo H, et al. Gene expression profiles of laser-captured adjacent neuronal subtypes. Nat Med 1999;5:117-22.

18 Sgroi DC, Teng S, Robinson G, et al. In vivo gene expression profile analysis of human breast cancer progression. Cancer Res 1999;59:5656-61.

19 Banks RE, Dunn MJ, Forbes MA, et al. The potential use of laser capture microdissection to selectively obtain distinct laser capture microdissection to selectively obtain distinct
populations of cells for proteomic analysis-preliminary populations of cells for proteomic analy

20 Schütze K, Lahr G. Identification of expressed genes by laser mediated manipulation of single cells. Nat Biotechnol 1998;16:737-42.

21 Fink L, Seeger W, Ermert L, et al. Real-time quantitative RT-PCR after laser-assisted cell picking. Nat Med 1998;4: 1329-33.

22 Dietmaier W, Hartmann A, Wallinger S, et al. Multiple mutation analyses in single tumour cells with improved whole genome amplification. Am f Pathol 1999;154:83-95.

23 Schermelleh L, Thalhammer S, Heckl W, et al. Laser microdissection and laser pressure catapulting for the generation of chromosome-specific paint probes. Biotechniques 1999; 27:362-7. 